\title{
Analysis of Factors That Influence Consumer Choice of Iron Deficiency Anemia Drugs
}

\author{
Gribkova E. I.* Galkina G.A. Pak T.V. \\ Peoples' Friendship University of Russia, Moscow, Russia \\ *Corresponding author. Email: Gribkova-ei@rudn.ru

\begin{abstract}
The aim of the study is to analyze factors that influence the choice of iron deficiency anemia drugs. The sociological survey was carried out in medical and pharmacy organizations. The survey involved 20 doctors who have used the drugs under study and 70 end randomly selected end users of drugs. All questionnaires were found to be valid. The following data were obtained: $66 \%$ of doctors prescribe iron supplements in combination with vitamins; $60 \%$ of doctors prefer imported drugs; $70 \%$ of respondents prescribe oral solutions; Actiferrin is prescribed in $88 \%$ of cases. The end consumers prefer domestic drugs. $58 \%$ of respondents answered that efficiency is the most important factor; for $90 \%$ of respondents, safety is important; $71 \%$ prefer Sorbiferr Durules, $70 \%$ prefer Maltoferr and other drugs.
\end{abstract}

Keywords: assortment analysis, iron deficiency anemia, consumer preferences, drug.

\section{INTRODUCTION}

To improve the quality of drugs, pharmacy organizations need to study preferences of their end and intermediate. Consumers [8-10]. The group of drugs used to treat iron deficiency anemia was selected due to the fact that iron deficiency anemia is the most common anemic syndrome it accounts for approximately $80 \%$ of all anemias.

\section{METHODS AND MATERIALS}

A comparative analysis of drugs used for treating iron deficiency anemia presented in Moscow pharmacies was carried out. In total, the survey involved 20 specialists working in medical organizations of Moscow.

A sociological study of doctors prescribing iron deficiency anemia drugs was carried out. The survey involved 20 Moscow doctors.

A sociological study of consumers was carried out in Moscow pharmacy organizations using a questionnaire to assess the demand for drugs. In total, 70 consumers were involved in the survey.

\section{RESULTS}

The results analysis technology included the following stages: (1) development of a database, which included iron deficiency anemia drugs registered in the Russian Federation and presented in Moscow pharmacies; (2) a sociological survey of doctors who had experience in prescribing these drugs; based on the data obtained, a positioning map for efficacy and safety was built; (3) a sociological study of consumers of drugs was carried out [1-4].
The database of iron deficiency anemia drugs made it possible to analyze them according to such characteristics as manufacturers, ATC-classification codes, dosage forms, etc. [5-7].

A list of manufacturers iron deficiency anemia drugs was created. The market share of domestic manufacturers is $23 \%$. It was found that the bulk of the Russian drugs is produced by PharmFirma Sotex. Figure 1 shows a list of Russian drug manufacturers (Figure 1).

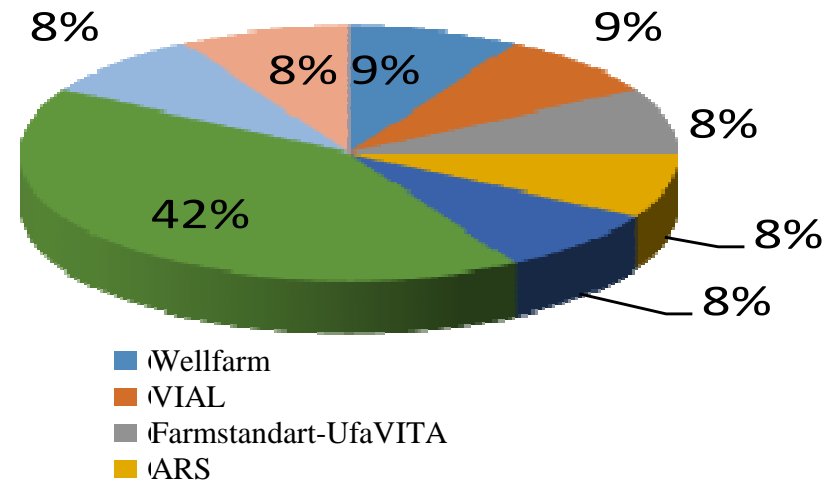

Figure 1 Russian manufacturers of iron deficiency anemia drugs

The analysis of iron deficiency anemia drugs according to the ATC classification showed that ferrum drugs predominate $(60 \%)$; they are followed by combinations of ferrum drugs + vitamin - $22 \%$; ferrum drugs + other drugs $-5 \%$; antianemic agent $-5 \%$; ferrum drugs + multivitamins $-4 \%$; antianemic agent $-4 \%$ (Fig. 2).

The drugs are represented by $58 \%$ of liquid medications, the share of solid forms is $48 \%$. An analysis of liquid drugs showed that solutions predominate $-68 \%$, syrups for oral administration accounted for $21 \%, 11 \%$ of 
all drugs were drops. When analyzing solid forms of drugs, the following results were obtained: tablets $-50 \%$, capsules $-40 \%$, pills $-5 \%$, plates $-5 \%$.

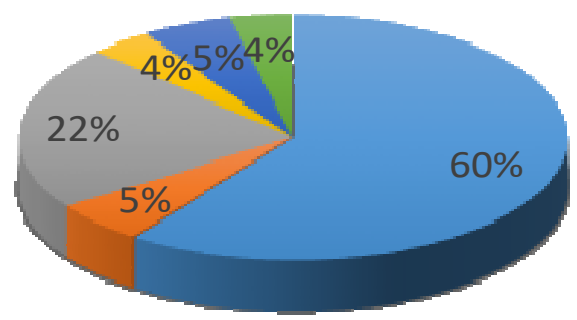

$$
\begin{aligned}
& \text { YFerrum drugs (FD) } \\
& X_{\mathrm{FD}}+\text { other drugs } \\
& \mathcal{X}_{\mathrm{FD}}+\text { vitamins } \\
& \mathrm{FD}+\text { multivitamins }
\end{aligned}
$$

Figure 2 Distribution of iron deficiency anemia drugs according to the ATC classification

In a sociological survey of doctors who have prescribed iron deficiency anemia drugs, 20 specialists met the following requirements: work experience of at least 10 years, higher education, advanced training courses taken not more than 2 years ago, experience of prescribing iron deficiency anemia drugs.

The doctors were asked to name the drugs they are prescribed for treating iron deficiency anemia. Their choices are shown in Figure 3.

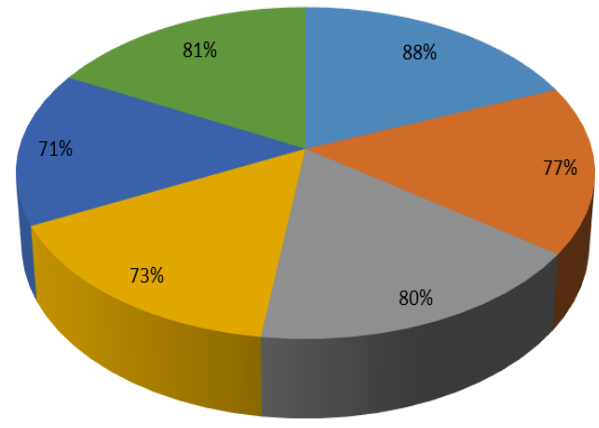

-Actiferrin

Sorbiferr Durules

Maltoferr

Ferroplex

"Ferrum Lek

-Likferr 1000

Figure 3 Drugs chosen by medical experts for treating iron deficiency anemia among medical experts

Analyzing the data obtained, it can be seen that Aktiferin is prescribed in $88 \%$ of cases, Likferr 1000 - in $81 \%$ of cases, Maltofer - in $80 \%$ of cases, Sorbifer Durules - in $77 \%$ of cases, Ferroplex - in $73 \%$ of cases, Ferrum Lek $-71 \%$ of cases.

The respondents were asked to assess their effectiveness and safety using a 10-point system. The assessment was carried out in accordance with the instructions for medical use: spectrum of indications, additional pharmacologically active substances, such as vitamins; dosage, possibility of taking it by children, etc. (positive characteristics); side effects, special conditions and instructions when taking these drugs, contraindications (negative characteristics). Each positive criterion was assigned +1 point, each negative criterion was assigned -1 point.

The result of the survey was a positioning map of efficiency and safety (Fig. 4).

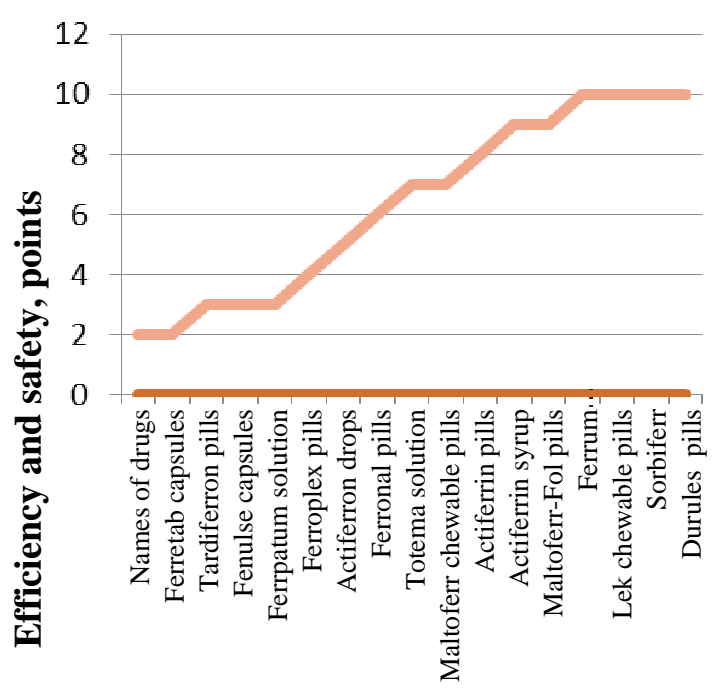

Names of drugs

Figure 4 Iron deficiency anemia drugs positioning map

On the positioning map, the leading positions were occupied by Sorbifer Durules pills (Hungary), Ferrum Lek chewable pills (Slovenia), Ferrum Lek syrup (Slovenia), Maltofer-Fall chewable pills (Switzerland), Maltofer syrup (Switzerland), Aktiferrin syrup (Israel). As a result, the drugs chosen by specialists were of foreign origin. The point assessment results are as follows:

1. Aktiferrin - "23"

2. Sorbifer - "15.5"

3. Maltofer - "2"

4. Ferroplex - "16.5"

5. Ferrum Lek - "0"

6. Likferr 1000 - "29"

Further, the price affordability was calculated in USD for each drug:
1. Aktiferrin- "10"
2. Sorbifer - "12"
3. Maltofer - "19"
4. Ferroplex - "3"
5. Ferrum Lek - "29"
6. Likferr 1000 - "46"

As a result of the study, the drugs that are most in demand by doctors were identified. Likferr 1000 scored the least points and the cost of one dose was the highest. This drug was an outsider. However, it should be noted that this drug has the requiredy dosage form (solution for 
intravenous administration and intramuscular injection). Maltofer turned out to be the optimal drug by the cost per dose and scores obtained.

It was also revealed that when drawing up a treatment regimen for iron deficiency anemia, most doctors $(80 \%)$ use combined treatment, only $20 \%$ of the doctors answered that they prescribe only one group of drugs from the ATX classifier: $66 \%$ of doctors prescribe ferrum drugs combined with vitamins; $61 \%$ - with other drugs; $58 \%$ with multivitamins; $55 \%$ - only iron supplements. Analyzing the data obtained, it was determined that $60 \%$ of doctors prefer imported drugs, $40 \%$-domestic drugs.

It was determined that $70 \%$ of doctors prefer solutions, $60 \%$ - tablets, capsules and pills, $59 \%$ - syrups and drops.

At the next stage, preferences of end users were studied. 70 people took part in the survey. The questionnaire consisted of two blocks. The first block was aimed at studying socio-demographic characteristics of end consumers (Fig. 5)

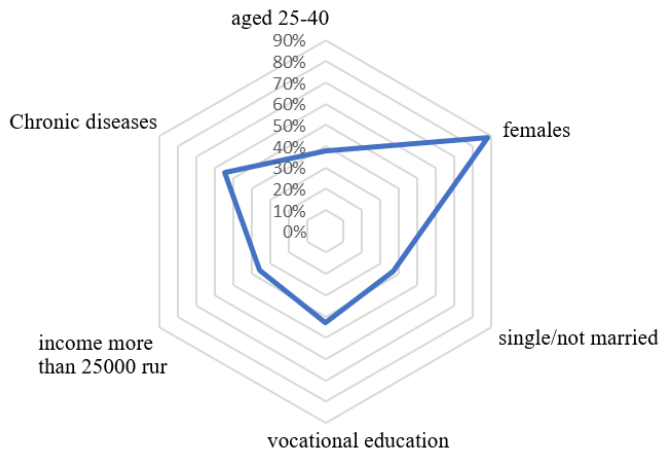

Figure 5 Portrait of an iron deficiency anemia drugs consumer

The second block consisted of questions that made it possible to determine consumer preferences in choosing the drugs and factors that influence their choice.

It turned out that $32 \%$ of the consumers receive information from doctors, $28 \%$ - from pharmacies, other respondents trust their friends $(13 \%)$, relatives $(15 \%)$, and the Internet (12\%). The majority of consumers $(90 \%)$ need advice of pharmacy employees, regardless of the source of information on the drug.

An analysis by country of production showed that $48 \%$ of the respondents prefer domestic drugs, $42 \%-$ imported drugs, and only $10 \%$ answered that the country of origin does not matter.

Analyzing the data on the preferred dosage form, we obtained the following results: $26 \%$ prefer pills, $23 \%$ prefer capsules, $20 \%$ prefer solutions, $18 \%$ prefer syrups, and $13 \%$ prefer drops.

When studying characteristics that are important for end users when choosing an iron deficiency anemia drug, we found that for $95 \%$ of respondents, the effectiveness is important, for $90 \%$ of respondents, safety is important; $83 \%$ answered that ease of use is important, and $75 \%$ answered that the price is also important.
We offered a list of drugs to indicate the preferred ones. $71 \%$ prefer Sorbiferr Durules, $70 \%$ - Maltoferr, $68 \%$ - Ferrum Lek, $65 \%$ - Likferr 1000, $61 \%$ Ferroplex, $58 \%$ - Aktiferrin (Fig. 6).
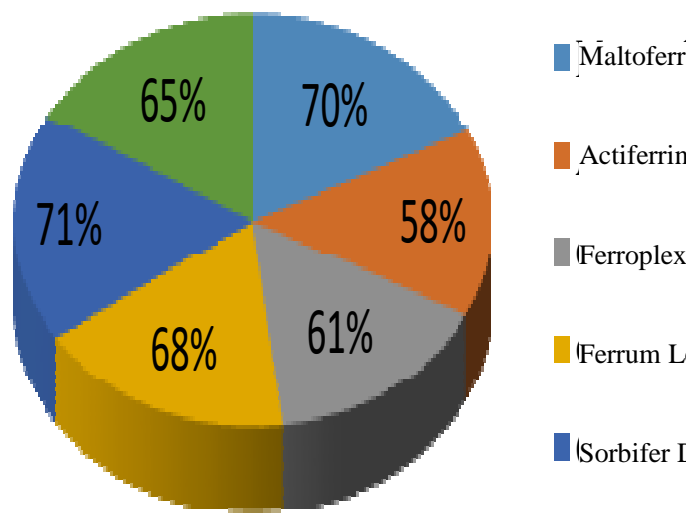

Ferrum Lek

ISorbifer Durules

.Likferr 1000

Figure 6 Consumer preferences when choosing iron deficiency anemia drugs

As a result, consumers prefer one of the drugs, which is among the leaders. This is Maltoferr.

\section{CONCLUSION}

The overwhelming majority of drugs used to treat iron deficiency anemia are ferrum drugs $(60 \%)$; drugs are produced in a liquid (58 \%) and solid (42\%) forms. Among solid forms, tablets $(50 \%)$ and capsules (40\%) predominate. Among liquid forms, solutions prevail (68\%).

During the survey of doctors, we obtained the following data: $66 \%$ of doctors prescribe iron supplements in combination with vitamins; $60 \%$ prefer imported drugs; $40 \%$ prefer domestic drugs; $70 \%$ prefer solutions, Aktiferin is prescribed in $88 \%$ of all cases.

The end consumers prefer domestic drugs (58\%), $95 \%$ of the respondents answered that the effectiveness is important when purchasing drugs; for $90 \%$ of the respondents, safety of the drug is important; $71 \%$ prefer Sorbifer Durules, $70 \%$ of the respondents answered that they prefer Maltoferr.

The results of the study will allow pharmacy organizations to optimize their assortment and improve quality of drugs.

\section{REFERENCES}

[1] A.V. Zozulev, Consumer Behavior, Textbook, Knowledge, Kiev, 2004, 364 p.

[2] V.V. Dorofeeva, V.D. Chan, Exploring the Factors Affecting Pharmaceutical Consulting in Vietnamese Pharmacies, Remedium, Magazine about the drug and med. equipment market, 4 (2019) 32-35. 
[3] I.V. Kosova, E.E. Loskutova, T.P. Lagutkina, V.V. Dorofeeva, A.A. Teodorovich, Economics and organization of pharmacy: textbook for student institutions of vocational education, Ed. by I.V. Kosova, Publ. Center Academy, Moscow, 2018, $448 \mathrm{p}$.

[4] O.A. Kulikova, L.I. Lavrentieva, O. V. Sokolova, Standardization of the process of serving the population in pharmaceutical organizations, Development, res. and market. of new pharmac. products: collection of sci. papers 69 (2014) 404-405, Pyatigorsk.

[5] M.M. Bahlol, T.P. Lagutkina, Analysis of scientific research on the promotion of pharmaceutical products in foreign countries: practical recommendations for pharmaceutical companies, Bull. of Siber. Med. 15(1) (2016) 60-68, Tomsk.

[6] S.A. Belanovsky Focus group method, Textbook, Nikolo-Media, Moscow, 2001, 280 p.

[7] T.P. Lagutkina, E.E. Loskutov, V.V. Dorofeeva, I.V. Kosova, A.A. Teodorovich, Pharmaceutical consulting as one of the means of implementing the concept of health literacy, Med. and pharmac. J. Pulse 1 (2020) 62-69, Kiev: TECHNOMED HOLDINGS.

[8] M.S Soboleva, E.E. Loskutova, I.V. Kosova, I.V. Amelina, Problems and the Prospects of Pharmaceutical Consultation in the Drugstores, Archives of Pharmacy Practice 11(2) (April - June 2020) 154-159.

[9] V.D. Tran, V.V. Dorofeeva, Using Q methodology in pharmacy research: a method overview and a pilot study, Article J. of Pharmac. Sci. and Res. 10(5) (2018) 1277-1280.

[10] E. Payne, CRM Guide: a path to improving customer management, Minsk, 2007, 384 p. 